\title{
Experimental demonstration of a suspended diffractively coupled optical cavity
}

\author{
M. P. Edgar, ${ }^{1, *}$ B. W. Barr, ${ }^{1}$ J. Nelson, ${ }^{1}$ M. V. Plissi, ${ }^{1}$ K. A. Strain, ${ }^{1}$ O. Burmeister, ${ }^{2}$ M. Britzger, ${ }^{2}$ \\ K. Danzmann, ${ }^{2}$ R. Schnabel, ${ }^{2}$ T. Clausnitzer, ${ }^{3}$ F. Brückner, ${ }^{3}$ E.-B. Kley, ${ }^{3}$ and A. Tünnermann ${ }^{3}$ \\ ${ }^{1}$ Department of Physics and Astronomy, University of Glasgow, Glasgow G12 8QQ, UK \\ ${ }^{2}$ Max-Planck-Institut für Gravitationsphysik (Albert-Einstein-Institut) and Institut für Gravitationsphysik, \\ Leibniz Universität Hannover, Callinstrasse 38, 30167 Hannover, Germany \\ ${ }^{3}$ Institut für Angewandte Physik, Friedrich-Schiller-Universität Jena, Max-Wien-Platz 1, 07743 Jena, Germany \\ *Corresponding author: m.edgar@physics.gla.ac.uk
}

Received July 10, 2009; revised September 3, 2009; accepted September 3, 2009; posted September 16, 2009 (Doc. ID 113921); published October 12, 2009

All-reflective optical systems are under consideration for future gravitational wave detector topologies. One approach in proposed designs is to use diffraction gratings as input couplers for Fabry-Perot cavities. We present an experimental demonstration of a fully suspended diffractively coupled cavity and investigate the use of conventional Pound-Drever-Hall length sensing and control techniques to maintain the required operating condition. (C) 2009 Optical Society of America

OCIS codes: 050.1970, 050.2230, 120.2230, 120.3180, 120.5700, 230.1950.

A network of first-generation laser interferometric gravitational wave detectors (LIGO, GEO600, VIRGO, and TAMA) is currently operating around the world [1]. All of these instruments are based on enhanced Michelson topologies utilizing several techniques to increase the stored laser power and hence the detector sensitivity, such as power recycling and optical cavities. Light is coupled in via partially transmissive mirrors, and the power in the substrates of some of the optical components such as coupling mirrors or beam splitters reaches or exceeds 1 $\mathrm{kW}$ at a wavelength of $1064 \mathrm{~nm}$. Proposed designs for next generation detectors require even higher circulating light levels to improve the detector sensitivity. Increasing the circulating power is troublesome, since a constant small fraction is absorbed in optical substrates. This causes localized heating that becomes progressively more serious because ever stronger thermal lenses are produced through the change in the refractive index with temperature [2]. A potential resolution to this problem is to use all-reflective optical components such as diffraction gratings to split and recombine light fields [3]. These devices are also of interest in other fields, for example, nonlinear optical cavity enhancement. A second motivation for the use of gratings in future interferometers arises if cryogenic techniques are required to reduce thermal noise. It is likely that materials that are opaque at $1064 \mathrm{~nm}$, such as silicon, will offer the best performance at cryogenic temperatures, and therefore either a nontransmissive mechanism must be found or the laser wavelength would need to be changed.

Although the study of interferometer topologies is essentially straightforward, dealing only with the simple relationships among linear light fields, numerical techniques are routinely used to compute the signals obtained at various photodetectors in response to changes in the relative positions of the optics. This is a requirement for the development of length sensing and control schemes required to keep the interferometers at the desired operating point and to read out the signals, including the gravitational wave signal. It is therefore important to understand how to model diffractive couplers and to validate such simulations by experiment.

Several proof-of-principle [4] and bench-top [5] experiments have been carried out to demonstrate the principles of operation for diffractive couplers in optical cavities. To examine the applicability of such systems to suspended instruments, one of the arm cavities of the Glasgow $10 \mathrm{~m}$ prototype interferometer was commissioned as a diffractively coupled optical cavity (see Fig. 1). Each of the optics in this system is suspended as a multistage pendulum to provide seismic isolation and allow freedom of motion. The diffraction grating under investigation was manufactured by etching a binary structure into a silica substrate, then coating with multiple alternating layers of $\mathrm{Ta}_{2} \mathrm{O}_{5}$ and $\mathrm{SiO}_{2}$ to give an ultra-lowloss-low-efficiency grating with a period of $d$ $=1450 \mathrm{~nm}$ [6]. The grating was mounted in the second order Littrow configuration, and illuminated at an angle of $47.2^{\circ}$ with $s$-polarized light at $1064 \mathrm{~nm}$ wavelength, from a $\mathrm{Nd}$ :YAG laser.

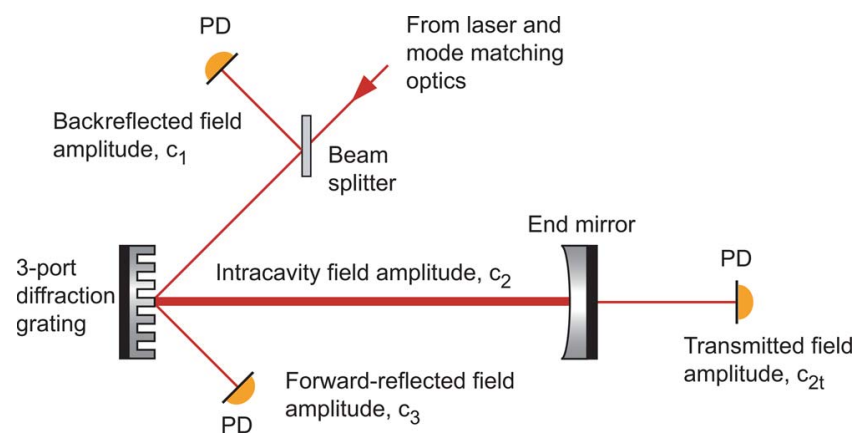

Fig. 1. (Color online) Simplified schematic of the threeport second-order Littrow mount grating used as the input coupler for a diffractive Fabry-Perot cavity. Tuned photodiodes (PDs) are positioned at all three output ports to detect the DC power and rf component for derivation of the control signals. 
The optical cavities within a gravitational wave detector must be maintained at the correct operating point, usually with the light from the laser resonant. To this carrier light, various modulation sidebands are added, e.g., as prescribed in the Pound-DreverHall (PDH) technique [7]. The light leaving the cavity is detected at output ports and information on the interaction between the frequency components can then be extracted by demodulating the detected signals with a local oscillator at the modulation frequency. As seen in Fig. 1 our system has three detection ports. To enable signal extraction from all three output ports, two different rf modulation frequencies were used. As with the normal PDH method, the choice of frequency for the forward-reflected and backreflected ports is not critical, provided that the sidebands are off resonance in the cavity when the carrier is resonant. A frequency of $10 \mathrm{MHz}$ was chosen for convenience. To obtain a nonvanishing signal from the transmitted port required the use of sidebands just off resonance, at $15.24 \mathrm{MHz}$, close to the free spectral range (FSR) of the cavity. The transmitted light can subsequently be detected on a tuned photodetector and demodulated to produce a symmetrical length sensing signal. The FSR of our cavity is $15.27 \mathrm{MHz}$ and, by monitoring the $\mathrm{DC}$ signal from the transmitted port, the corresponding FWHM was determined to be $13.80 \pm 0.64 \mathrm{kHz}$.

The operation of a traditional two-port FabryPerot cavity is determined by the reflection/ transmission efficiencies of the mirrors. However, utilizing a three-port diffraction grating as an input coupler to a Fabry-Perot cavity, the optical field interactions become more complex. The scattering matrix of a three-port grating can be represented by

$$
\mathbf{S}_{3 p}=\left[\begin{array}{ccc}
\eta_{2} e^{i \phi_{2}} & \eta_{1} e^{i \phi_{1}} & \eta_{0} e^{i \phi_{0}} \\
\eta_{1} e^{i \phi_{1}} & \rho_{0} e^{i \phi_{0}} & \eta_{1} e^{i \phi_{1}} \\
\eta_{0} e^{i \phi_{0}} & \eta_{1} e^{i \phi_{1}} & \eta_{2} e^{i \phi_{2}}
\end{array}\right],
$$

where $\eta_{0,1,2}$ and $\phi_{0,1,2}$ are the amplitude diffraction efficiencies and the phase changes on the diffraction for zeroth, first, and second orders, respectively, and $\rho_{0}$ is the amplitude reflectivity at normal incidence. If these values are known along with the reflectivity/ transmittivity efficiencies $\left(\rho_{1} / \tau_{1}\right)$ of the end mirror, then all properties of the cavity can be determined. The input-output relations for a three-port diffraction grating coupled Fabry-Perot cavity have been investigated [8] and yield amplitudes for the fields at each port described by

$$
\begin{gathered}
c_{1}=\eta_{2} e^{i \phi_{2}}+\eta_{1}^{2} e^{2 i\left(\phi_{1}+\phi\right)} d, \\
c_{2 t}=i \tau_{1} \eta_{1} e^{i\left(\phi_{1}+\phi\right)} d, \\
c_{3}=\eta_{0}+\eta_{1}^{2} e^{2 i\left(\phi_{1}+\phi\right)} d,
\end{gathered}
$$

where $\phi$ represents the phase acquired after one round trip of the cavity and the resonance factor is defined by $d=\left[1-\rho_{0} \rho_{1} e^{2 i \phi}\right]^{-1}$.

Based on these field equations a numerical simulation of the diffractive cavity was built using MATLAB to allow a comparison between modeled predictions and experimental results. Using grating parameters provided by the Albert-Einstein-Institute in Hannover and the specifications of the end mirror from the manufacturer, the light field amplitudes and the power at each port could be calculated. Direct measurement of the average light power at each output port showed agreement to within $12 \%$ of the model. The calculated finesse of the cavity is then $1177 \pm 27$, which is in good agreement with the measured finesse of $1107 \pm 51$.

One way to establish whether the sensing signals predicted by the model agree with the experiment is to sweep the length of the cavity by at least one round-trip wavelength (i.e., one FSR). Then the demodulated signal from each port can be compared with the prediction. The slope of this signal at the operating point gives the response for that output port.

The rf signal from each detection port can be demodulated in two orthogonal phases, called "in phase" and "quadrature phase" for maximum and minimum responses, respectively. The demodulated signals obtained in the experiment are shown in Fig. 2 and are seen to be in good qualitative agreement with modeled predictions. The discrepancies between the experiment and the modeled signals can be attributed to the finite rate of sweep in the experiment.

To calibrate the demodulated signals detected at all three ports, the relative size of the in-phase slopes was compared with the slope of the signal from the
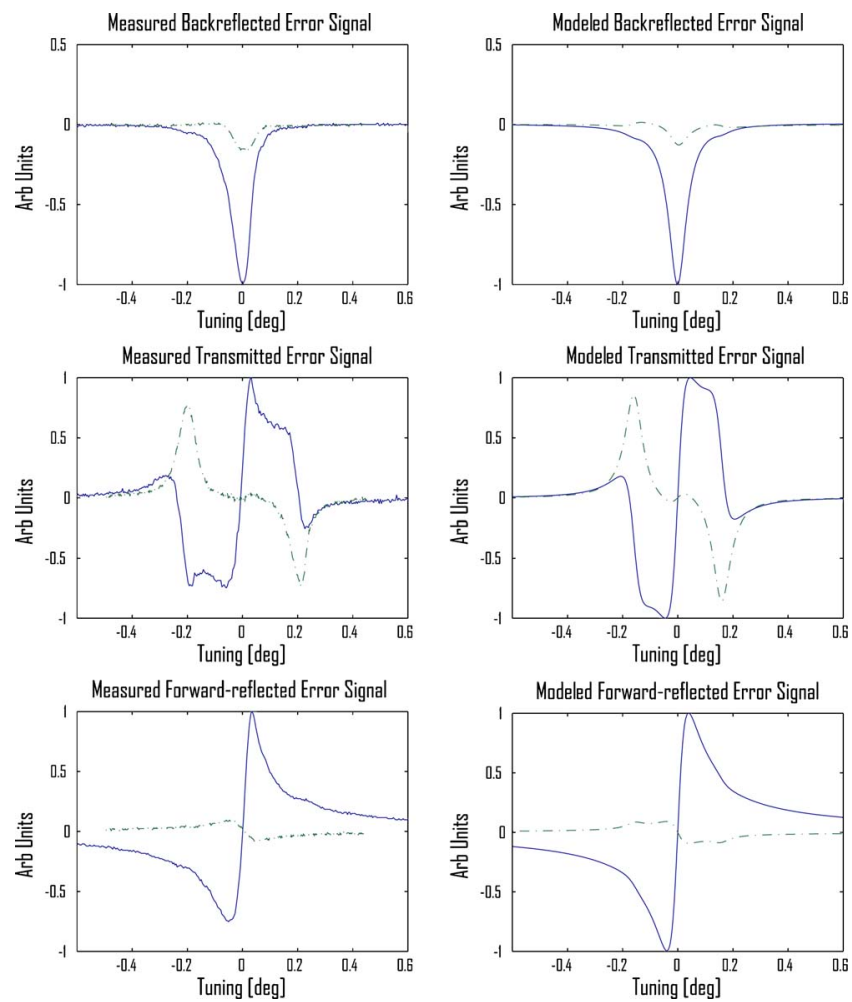

Fig. 2. (Color online) Normalized rf power for all three output ports $C_{1}$ (top), $C_{2}$ transmitted through the end mirror (middle), and $C_{3}$ (bottom). The solid (blue) trace indicates in-phase measurements, and the dashed (green) trace indicates quadrature phase. The absolute scaling between modeled ports 1,2 , and 3 is 2:1:163. 
transmitted port. The ratios of the signal responses are presented in Table 1, indicating a good quantitative agreement with the model.

Having demonstrated the validity of the model for this experimental configuration, the numerical simulation can be extended to investigate a more general case. In particular, previous experimental work with tabletop cavities [5] had demonstrated an asymmetric behavior of the reflected carrier power levels. The extent of the asymmetry was seen to be determined by the values of the $\eta_{0}$ and $\eta_{2}$ diffraction efficiencies. With this in mind, we adapted the numerical simulation to investigate the effects of asymmetry on the demodulated output signals with grating parameters chosen to accentuate the observable effects.

It is clear from the modeled traces (see Fig. 3) that the demodulated signals from the forward-reflected and backreflected ports do exhibit asymmetry as expected. However, through a careful choice of demodulation phase, we can extract signals from each of these ports, which sum together to exactly reconstruct a traditional PDH locking signal. Additionally, while the asymmetry is clearly observable for our exaggerated case, the demodulated signals from the forward-reflected and backreflected ports will always exhibit some asymmetry - this is limited by the fact that $\eta_{0}$ and $\eta_{2}$ have a lower bound owing to energy conservation [8].

In our experimental configuration the cavity was locked using the transmitted error signal, which exhibits symmetrical behavior around the center of resonance. The ability to lock at this known operating point means that we can use the diffractively coupled cavity to investigate the dynamic effects associated with suspended optical systems. In particular, when dealing with diffractive couplers, phase shifts owing to translational motion of the coupler relative to the laser beam are predicted to introduce additional noise when compared with an equivalent traditional cavity configuration [9]. Future experiments on this suspended diffractive system will probe the effect of translational grating motion and associated effects owing to cavity misalignment.

In conclusion, our experimental results confirm the theoretical foundations supporting a diffractively coupled Fabry-Perot cavity and successfully demonstrate the use of all-reflective optics in a fully suspended environment. We have developed a numerical model for length sensing and control signal extraction from a diffractively coupled cavity based on the conventional rf sideband modulation and demodula-

Table 1. Measured and Modeled Signal Responses

\begin{tabular}{lcc}
\hline & $\begin{array}{c}\text { Measured } \\
\text { Value } \\
(\mathrm{dB})\end{array}$ & $\begin{array}{c}\text { Modeled } \\
\text { Value } \\
(\mathrm{dB})\end{array}$ \\
\hline Barameter & -26.93 & -22.51 \\
Transmitted/transmitted & 0 & 0 \\
Forward-reflected/transmitted & 43.07 & 44.74 \\
\hline
\end{tabular}

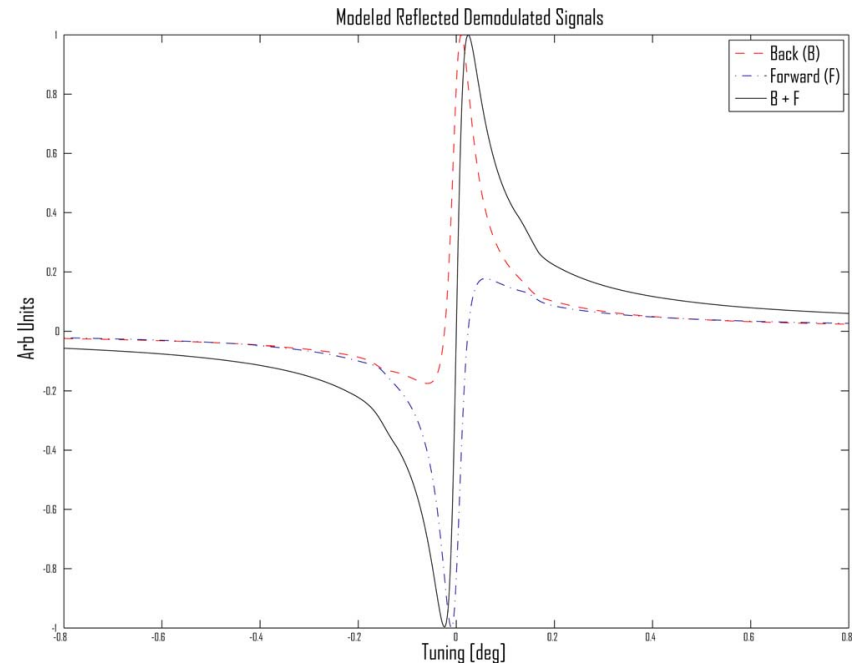

Fig. 3. (Color online) Simulated reflected field, demodulated signals $C_{1}$ (red dashed trace) and $C_{3}$ (blue dasheddotted trace), and the combined demodulated signal $C_{1}$ $+C_{3}$ (black solid trace). The grating parameters chosen here are for an ideal (lossless) grating with $\rho_{0}=0.99663$, $\eta_{1}=0.0407$, and $\eta_{0}=\eta_{2}=0.7065$.

tion techniques, and we have shown that the system is well understood through both quantitative and qualitative experimental verifications. Based on these findings we have also observed the effect of grating parameters to signal extraction from a diffractively coupled system by utilizing the principles of energy conservation. The framework for signal extraction from a suspended diffractive system is now well understood.

This work was supported by the University of Glasgow and the Science and Technology Facilities Council. The authors would also like to acknowledge the Sonderforschungsbereich TR7 of the Deutsche Forschungsgemeinschaft (DFG) for financial support.

\section{References}

1. P. Aufmuth and K. Danzmann, New J. Phys. 7, 202 (2005).

2. K. A. Strain, K. Danzmann, J. Mizuno, P. G. Nelson, A. Rüdiger, R. Schilling, and W. Winkler, Phys. Lett. A 194, 124 (1994).

3. A. Bunkowski, O. Burmeister, P. Beyersdorf, K. Danzmann, R. Schnabel, T. Clausnitzer, E.-B. Kley, and A. Tünnermann, Opt. Lett. 29, 2342 (2004).

4. K.-X. Sun and R. L. Byer, Opt. Lett. 23, 567 (1998).

5. A. Bunkowski, O. Burmeister, K. Danzmann, R. Schnabel, T. Clausnitzer, E.-B. Kley, and A. Tünnermann, Opt. Lett. 31, 2384 (2006).

6. T. Clausnitzer, E.-B. Kley, A. Tünnermann, A. Bunkowski, O. Burmeister, K. Danzmann, R. Schnabel, S. Gliech, and A. Duparré, Opt. Express 13, 4370 (2005).

7. R. W. P. Drever, J. L. Hall, F. V. Kowalski, J. Hough, G. M. Ford, A. J. Munley, and H. Ward, Appl. Phys. B 31, 97 (1983).

8. A. Bunkowski, O. Burmeister, K. Danzmann, and R. Schnabel, Opt. Lett. 30, 1183 (2005).

9. A. Freise, A. Bunkowski, and R. Schnabel, New J. Phys. 9, 433 (2007). 